\title{
Liquefied extinguishing agent discharge to an overpressure-sensitive enclosed volume
}

\author{
Lukáš Hurda ${ }^{1, *}$, and Richard Matas ${ }^{1}$ \\ ${ }^{1}$ University of West Bohemia in Pilsen, New Technologies - Research Centre, Univerzitní 8, Pilsen, \\ Czech Republic
}

\begin{abstract}
The throttling of liquefied substances from high pressure vessels to an enclosed volume starting at atmospheric pressure is described in order to determine thermodynamic state of the extinguished room gaseous contents. Time dependent, $0 \mathrm{D}$ mathematical model is implemented describing the state inside the agent container, the isenthalpic throttling in the distribution system, agent vaporization and mixing with air. The agent is modelled as real gas. Other influences on the process including the heat transfer from selected solid parts inside the room and the gas mixture leakage out of the room are taken into account. Main outcome is an MS Excel tool for integrated fire extinguishers design optimization. The optimization balances the two contradictory requirements: Agent volumetric concentration to sustain the fire extinguishing capabilities and tolerable room overpressure. Agent fill weight and discharge time are being adjusted. The discharge time is controlled by the distribution piping and spray nozzles design. System operation is checked concerning various initial and boundary conditions.
\end{abstract}

\section{Introduction}

The design apparatus for integrated fire extinguishing system is described. Many aspects of the process are covered by standards, but the need to be worried about the emerging overpressure in the extinguished volume asks for careful analysis and proposal of new techniques.

The thermodynamic system of interest consists of two main parts: an enclosed volume (a room) and extinguishing agent container (a pressure vessel).

The room is initially filled with clean, dry air at atmospheric or near-atmospheric pressure and arbitrary temperature. The heat transfer between walls and its surroundings is omitted completely as the real-life object being modelled is well insulated and relatively low temperature gradients are expected. The model is not completely closed speaking of mass conservation, a simple model of gas mixture leakage based on the pressure rise or fall during extinguishing agent discharge is implemented.

The vessel may have different initial temperature than the room. The heat transfer through the vessel wall is also neglected as the conditions for its evaluations are unclear. The vessel is mounted inside the room, but it is covered in a way that prevents immediate

\footnotetext{
*Corresponding author: hurda@ntc.zcu.cz
} 
propagation of temperature changes of room gaseous contents to the nearest surroundings of the vessel. Another argument for neglecting the heat transfer is that it should not have a significant effect on the discharge process from the energetic point of view - the energy from air reaches the agent either through the vessel wall or through direct contact after its discharge.

There is a piping system ending with nozzle sets for agent distribution installed in the room. This parts' heat capacity and heat transfer properties are neglected due to similar reasons as for the vessel. The whole distribution system is modelled only in terms of pressure drop.

Three substances' real gas thermodynamic data from the NIST REFPROP database are in use for various calculations of agent state change: $\mathrm{CO}_{2}$ and two hydrocarbon/halocarbon mixtures. These mixtures are used for approximating a pure compound used in reality that is not available in properties database. The substitutes were chosen based on their interchangeability in refrigeration applications as stated in [3]. The agent is expected to be liquid initially.

The fire extinguishing system proper function is ensured by sufficient volumetric concentration of agent in the extinguished volume, it is checked throughout the timedependent calculation. The unusual limit of overpressure comes from the demand for integrity of lightweight structural design of the room walls.

\section{Thermodynamic state of extinguishing agent in the pressure vessel}

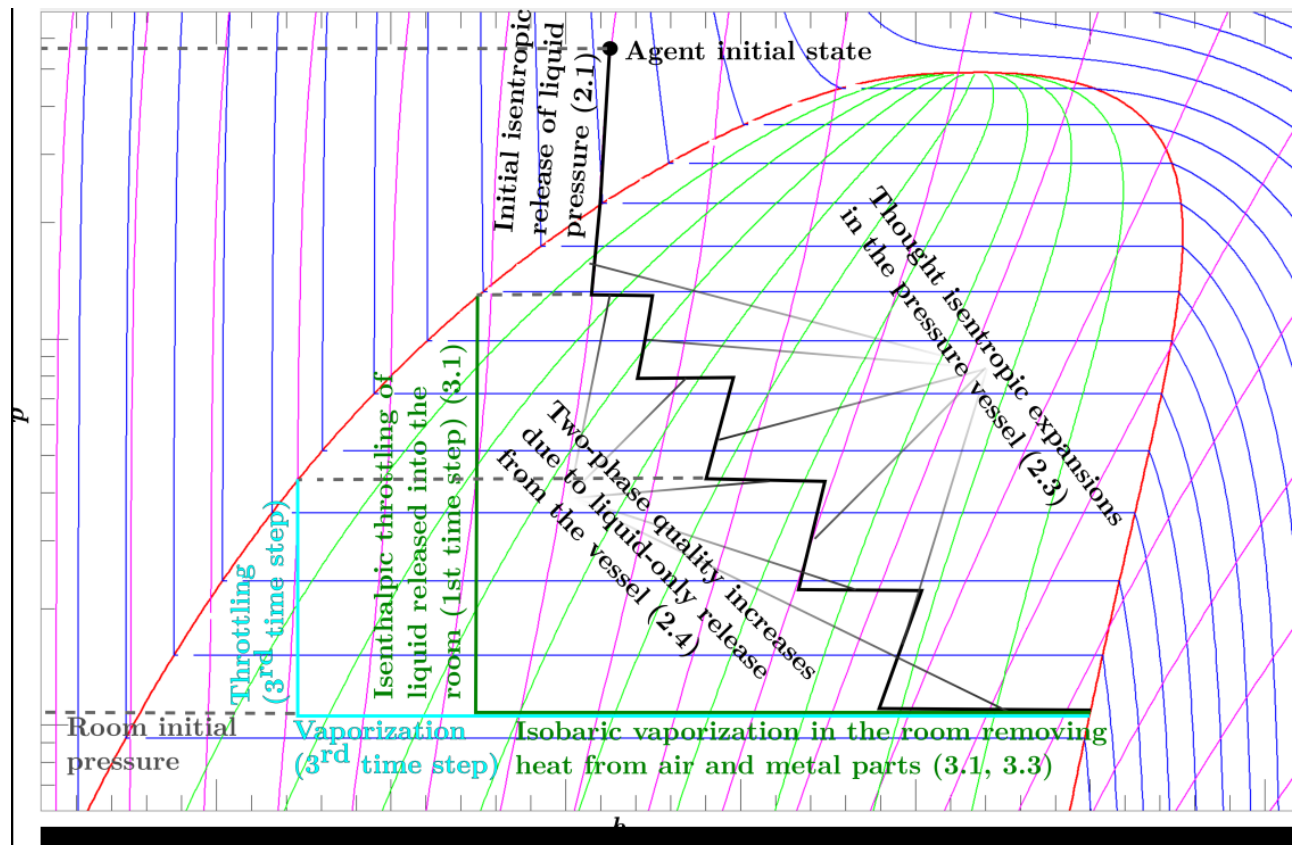

Fig. 1. A schematic pressure-enthalpy diagram of the extinguishing agent with annotated examples of phenomena described in $(2,3)$. Red - Saturation curves; blue - isotherms; magenta - isentropes, green - curves of constant two-phase quality. Other curves annotated in the figure by text of corresponding colour. (Own work based on NIST REFPROP fluid thermophysical database program).

The main reason why to be concerned with the process going on during emptying of the bottle is to get the starting point of isenthalpic expansion (throttling) which occurs in the piping and mainly nozzles (see (3.1)). Like that, the enthalpy of the released agent is determined. 
The vessel is designed to release only the liquid phase of the agent, even though the agent enters the two phase region just after the start of release. This is ensured by siphon tube or bottom release socket. The presence of relatively well separated liquid and vapour phases is expected, despite the ongoing boiling process in the vessel.

This means that the state of the released agent is to be found on the liquid saturation curve. It also needs to be examined how the agent pressure and mass drops with time. After that, the state of agent in the bottle can be determined in discrete time levels.

\subsection{Initial release of pressure in fluid}

In the first moment after container socket is opened, the pressure drops quickly as the contents of the container are mostly incompressible. In reality, it is necessary for the vessel to be filled with a small fraction of inert gas to compensate for the temperature changes of the vessel contents, but this fraction occupies a small volume to have a significant effect on the pressure release.

According to the statement above, the first time step of the calculation is finding the point on the liquid saturation curve which is reached by isentropic expansion of fluid from the prescribed initial pressure and temperature, see (1). The isentropic nature is expected as the kinetic aspects of the process are minor.

$$
p_{\text {sat }}=f\left(s_{\text {sat }}\right) ; s_{\text {sat }}=s_{\text {init }}=f\left(p_{\text {init }}, T_{\text {init }}\right)
$$

\subsection{Mass flow estimation}

An important assumption is made that the mass flow rate is constant during most of the agent discharge process. This conforms with $[1,2]$. The value of the mass flow rate is in fact the input quantity for the design of the distribution system, so it is not calculated in any way, but may be a subject of optimization.

Verification of the piping and nozzles design was a subject to experiment. In Figure 2, a constant mass flow rate may be observed for the most of the total mass of agent discharged. The diminishing outflow on the right side of Figure 2 is the part of discharge after "end of liquid" point. This point is reached extremely early in case of CO2 test due to reaching the states under the triple point, where the vessel siphon tube freezes full. This later discharge is neglected in the calculation and the discharge is considered to continue at the prescribed constant flow rate.

An explanation why the two phase, boiling mix discharge rate does not vary with dropping pressure in the container may lie in the subsonic design of the spray nozzles and the fact that pure liquid is entering the distribution system.

Inarguably, the fluid becomes a two phase mixture before reaching the nozzles, but the density of the mixture is probably close to constant. The boiling process in the piping determines the resulting density. It should be dependent only on the time a particle of fluid spends in the pipe and the parameters of the heat transfer through the pipe wall - these are believed to vary few.

The pressure ratio is high enough to choke the flow through the convergent nozzles for the most part of gas release. The critical pressure ratio ranges between $0.55 \div 0.58$ for all subjected gases.

The end of the discharge is determined by the point where there is only vapour and no liquid in the vessel. The neglected short period of subsonic outflow could possibly start before or after this point. 


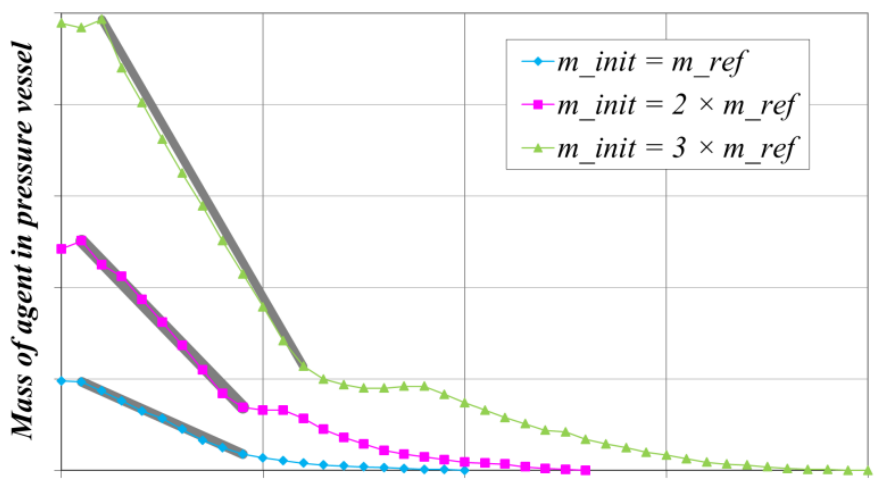

Time

Fig. 2. The discharge process for three experimental tests on a room prototype with $\mathrm{CO}_{2}$ used for verification of the constant mass flow rate. Thick grey lines indicates the linear approximation of mass, i.e. approximation of constant mass flow rate.

\subsection{Isentropic expansion in two-phase region}

The first of the two processes modelling the liquid release from the vessel mimics the expansion of the remaining contents of the vessel. The concurrent increase of two-phase quality is described in the next subsection.

The total volume of interest is obtained from total mass of agent and specific volume of saturated liquid in a state after reaching saturation as described in 2.1. The known volume of the container is not taken into account to dismiss the influence of inert gas vessel fill.

As the mass flow rate is known, the released mass per every time step is determined and the related volume too. This released volume is taken as the total volume change for the analysed isentropic expansion. The specific volume after the thought expansion can then be determined as in (2):

$$
v_{e n}=\frac{V+\Delta m_{e} \cdot v_{e(n-1)}}{m_{e}}
$$

The state is then determined by $s_{n}^{e}, v_{n}^{e}$, but as the shapes of isochoric and isentropic curves are not known explicitly in two phase region, a numerical solution is made rather than curve reconstruction. The pressure and temperature after expansion is found from table based on the equality of quality evaluated from entropy and from specific volume (note the indices $s, v$ for respective quantities) (3), .

$$
x_{e n s}=\frac{s_{e n}-s_{l i q}}{s_{v a p}-s_{l i q}} \quad x_{e n v}=\frac{v_{e n}-v_{l i q}}{v_{v a p}-v_{l i q}}
$$

Then, the pressure and temperature is found at which the condition $x_{e n s}-x_{e n v}=0$ is met. And then $x_{e n s}=x_{e n v}=x_{e n}$.

\subsection{State change due to liquid release}

As the total volume increase discussed in previous subsection is only thought, the mass decrease in the pressure vessel is further modelled. An increase in two-phase quality at constant pressure and temperature is calculated. Only liquid is released thanks to siphon tube or bottom socket of the vessel. 
The relation of the quality after thought expansion (pre-release) to the real quality after the concurrent liquid release and expansion is expressed simply in (4).

$$
x_{e n}=x_{e n \text { pre-release }} \cdot \frac{m_{n-1}}{m_{n}}
$$

This practice is legit as long as the time step is short enough, so the increase of quality is small. This can be possibly measured by the difference of the achieved fluid state from the presented calculation and if the expansion is calculated after the liquid release - in reverse order than presented. The proposed order was chosen to avoid the singular behaviour of quality increase calculation from saturated liquid state, where $x=0$.

\section{Thermodynamic state of gas mixture in the room}

\subsection{Released agent temperature and enthalpy}

The phenomena occurring during the flow of extinguishing agent from the pressure vessel through piping to nozzles in the room is believed to be an isenthalpic expansion, throttling. Therefore, the initial state for the expansion of liquid agent for each time step of the calculation is found on the left saturation curve at current pressure and temperature in the vessel.

It expands to the pressure present in the room and a temperature bound to this pressure in the two phase region. Gas is always in two phase state of different quality and the temperature is usually lower than the room contents.

The amount of heat for complete evaporation of the agent is expected to be transferred rapidly from the current gaseous contents of the room. The portion of the specific enthalpy of state change can be evaluated as follows (5):

$$
q_{\text {evap }}=h_{\text {d n vap }}-h_{d n}
$$

The temperature of the room contents before the mixing calculation is initiated is lowered by the heat consumed by the phase change of the agent as described in (6).

$$
T_{n \text { r premix }}=T_{r(n-1)}-\frac{q_{\text {evap }} \cdot \Delta m}{c_{(n-1) r} \cdot m_{(n-1) r}}
$$

\subsection{Mixing process}

The mixing of the discharged agent with the gaseous contents of the room is estimated with the theory of ideal gas. In the first time step, pure air is mixed with agent and the successive steps are calculated considering the already existing mixture. The agent is considered to be in saturated vapour state, usually colder than the rest of the room.

The specific heat and specific gas constant of the mixture is a mass weighted average. The temperature is an average weighted by mass and specific heat of the components.

\subsection{Heat addition from metal bodies}

When the room cools down rapidly, some parts may be able to expel its heat content as well as the gases do. There are a few factors to assess the ability to expel heat rapidly. Bodies worth this analysis need to be made of high conductive materials, have thin walls and large surface area exposed directly to the air. Also the total amount of heat per Kelvin (mass times specific heat) in such bodies must be high enough to have any impact on the 
whole process of interest. The eligible parts of room interior are sheet metal and thin metal profiles.

The presented method is very simple, but it was verified by more complex apparatus and has shown good agreement. The temperature distribution in the profile is neglected. A single value of bulk temperature is used. For the initialization of an iterative process, the metal part temperature is expected not to change from the previous time step (7).

$$
T_{m \text { ninit }}=T_{m(n-1)}
$$

Only two iterations of the process presented in (8), (9) give satisfactory results.

$$
\begin{gathered}
Q_{m n}=\alpha \cdot A_{m} \cdot\left(\frac{T_{m(n-1)}+T_{m n}}{2}-T_{n r}\right) \\
T_{m n}=T_{m(n-1)}-\frac{Q_{m n}}{c_{m} \cdot m_{m}}
\end{gathered}
$$

A qualified guess has to be made for the heat transfer coefficient $\alpha$ estimation. It may lie somewhere between $10 \div 50 \mathrm{~W} /\left(\mathrm{m}^{2} \mathrm{~K}\right)$. It can be adjusted concerning the exposure of the metal surfaces to the nozzle wake areas, a possible contact with liquid particles of the agent and other factors.

\subsection{Control volume leakage}

Some slots and chinks may be present in the outer bounds of the room structure. When an overpressure between the room and its surroundings occurs during the fire extinguishing process, the gases may leak out of the room. This may have a positive effect of the dropping overpressure.

The amount of leak per time step of calculation is based on current overpressure, the surroundings are considered to stay at constant pressure equal to the initial room pressure. Due to low discharge velocities, incompressible gas dynamics theory is adopted. The discharge velocity is expressed simply as $w=\sqrt{\Delta p \cdot v_{r}}$. The calculated velocity reaches about $90 \mathrm{~m} / \mathrm{s}$. The Mach number of the gas close to regular atmospheric conditions is then reaching a maximum of about 0.3. Under the circumstances of approximations already made, such flow could be considered incompressible.

The equivalent area of the ideal nozzle $A$ is estimated from an experiment carried out at reference conditions. The leaked mass at current time step is then $m_{l}=\Delta t \cdot w / v_{r}$.

\subsection{Resulting overpressure and volumetric concentration}

The overpressure between the room and the surroundings is expressed by perfect gas equation of state (10). The mass here is the mass of agent added to the mass of initial air content in the room and the leaked mass is subtracted. The specific gas constant and mixture temperature is discussed in 3.2. The volume of the room is a predefined constant.

$$
p_{r}=\frac{m_{r} r_{r} T_{r}}{V_{r}}
$$

\section{Conclusions}

A set of coherent calculations was built together for the purpose of integrated fire extinguishing system design and analysis of various operating conditions. The overpressure in the extinguished room is checked throughout the time dependent calculation. 
There are a few key parts of the work besides many trivial calculations.

The first is the assumption of constant mass flow rate of the extinguishing agent into the room, which is inevitable, but it is not causing inaccuracies as it is close to reality. The assumption is based on both external references and an experimental results.

The second is the thorough decomposition of the thermodynamic processes and the decisions on which way to model them. A summary is given in Figure 1. Many issues has been overcome on the side of using REFPROP tables imported as raw real gas data into MS Excel.

Last but not least, the identification of significant influences is made. The heat of metal bodies and control volume leakage (chapters $3.3,3.4$ ) are incorporated into the calculation.

According to the preliminary experiments made on the room prototype, there may be other influences on the process. The walls are not rigid, so the overpressure causes deformation resulting in enlargement of the total room volume. The gases may also loose significant amount of internal energy due to the work being done on the walls deformation. Analysis of those phenomena may lead to the future enhancements of the design tool.

The results were developed in the specific research (ZCU SGS-2016-045).

\section{Nomenclature}

$\begin{array}{lll}c \text { - specific heat (isobaric) } & {[\mathrm{J} / \mathrm{kg} / \mathrm{K}]} \\ \mathrm{m} \text { - mass } & {[\mathrm{kg}]} \\ Q \text { - total heat } & {[\mathrm{J}]} \\ r \text { - specific gas constant } & {[\mathrm{J} / \mathrm{kg} / \mathrm{K}]} \\ T \text { - absolute temperature } & {[\mathrm{K}]} \\ V \text { - volume } & {\left[\mathrm{m}^{3}\right]} \\ w \text { - flow velocity } & {[\mathrm{m} / \mathrm{s}]} \\ \alpha \text { - heat transfer coefficient } & {\left[\mathrm{W} / \mathrm{m}^{2} / \mathrm{K}\right]}\end{array}$

$h$ - specific enthalpy $\quad[\mathrm{J} / \mathrm{kg}]$

$p$ - absolute pressure $\quad[\mathrm{Pa}]$

$q$ - heat per kilogram mass $[\mathrm{J} / \mathrm{kg}]$

$s$ - specific entropy $\quad[\mathrm{J} / \mathrm{kg} / \mathrm{K}]$

$t$ - time $\quad[s]$

$v$ - specific volume $\quad\left[\mathrm{kg} / \mathrm{m}^{3}\right]$

$x$ - two-phase quality $\quad[-]$

Indices:

d - agent discharge state

init - initial

liq - liquid

n - time step number

$r \quad$ room

$\begin{array}{ll}\text { e } & \text { - extinguishing agent state } \\ 1 & \text { - leak } \\ \text { m } & \text { - metal } \\ \text { vap } & \text { - vapour } \\ \text { sat } & \text { - saturation state }\end{array}$

\section{References}

1. M. Ahmad, L. Buit, O. Florisson, C. Hulsbosch-Dam, M. Bögemann-van Osch, M. Spruijt, F. Davolio, Experimental Investigation of CO2 Outflow from a Highpressure Reservoir, Energy Procedia, 37, 3005-3017, ISSN 1876-6102, http://www.sciencedirect.com/science/article/pii/S187661021300430X, (2013)

2. National Fire Protection Association, NFPA Code 12A: Standard on Halon 1301 fire extinguishing systems ISBN: 978-145591885-0, https://www.nfpa.org/codes-andstandards/all-codes-and-standards/list-of-codes-and-standards/detail? code=12A, (2018)

3. Linde AG, Retrofit Guide, https:/www.linde-gas.com/internet.global.lindegas.global/en/images/Refrigerant\%20Retrofit\%20guides17_109809.pdf? $\mathrm{v}=5.0$, (2017) 